\title{
Indirect Principle Analysis of the Kinematic Errors in Five-Axis Machine Tool Rotary Table Based on the Error Sensitive Direction Vector
}

\author{
Ya Zhang ${ }^{1, a^{*}}$, Lin Zhang ${ }^{2, b}$ \\ ${ }^{1}$ Zhejiang University of Science and technology, Hangzhou, 310027, China \\ ${ }^{2}$ Yangzhou Polytechnic College, Yangzhou, 225127, China \\ E-mail: ayazhang1982@126.com, b330213049@qq.com
}

Keywords: Machine tool, Kinematic errors, Indirect measurement

\begin{abstract}
The kinematic errors of five-axis machine tool can be identified in no-cutting situation by the special instruments. However, the measurement process is complex and time-consuming. Machining paths of the test piece can represents the relationship of the kinematic errors of the rotary axis and the machining errors of the test piece. A series of machining paths were proposed to identify the kinematic errors. A equation set of the kinematic errors was set up, from which the kinematic errors can be solved.
\end{abstract}

\section{Introduction}

The kinematic errors of five-axis machine tool can be identified by the special instruments, such as double ball bar ${ }^{[1-5]}$, R-test ${ }^{[6,7]}$. However, these methods have the disadvantages of computational complexity, high cost, low universality, and so on. Based on the machining tests, this paper proposed the concept of virtue sensitive direction vector, and designed a series of machining tests. The kinematic equations set of the machining errors can be obtained and three kinematic errors of the tilt axis of the rotary table can be identified.

\section{Kinematic error identification principle based on the machining tests}

The kinematic model of the rotary table is shown in Fig.1. The kinematic errors of the rotation axis (C-axis) can be separated from the similar machining tests when the tilt axis (A-axis) is stationary ${ }^{[8]}$. Therefore, in the kinematic chain, only the translation from the coordinate $\{C\}$ to $\{A\}$ and the translation from the coordinate $\{\mathrm{A}\}$ to $\{\mathrm{F}\}$ should be considered ${ }^{[9]}$, as follows:

$$
{ }_{A}^{F} R=\left[\begin{array}{ccc}
C \alpha & -S \alpha & 0 \\
S \alpha & C \alpha & 0 \\
0 & 0 & 1
\end{array}\right]
$$




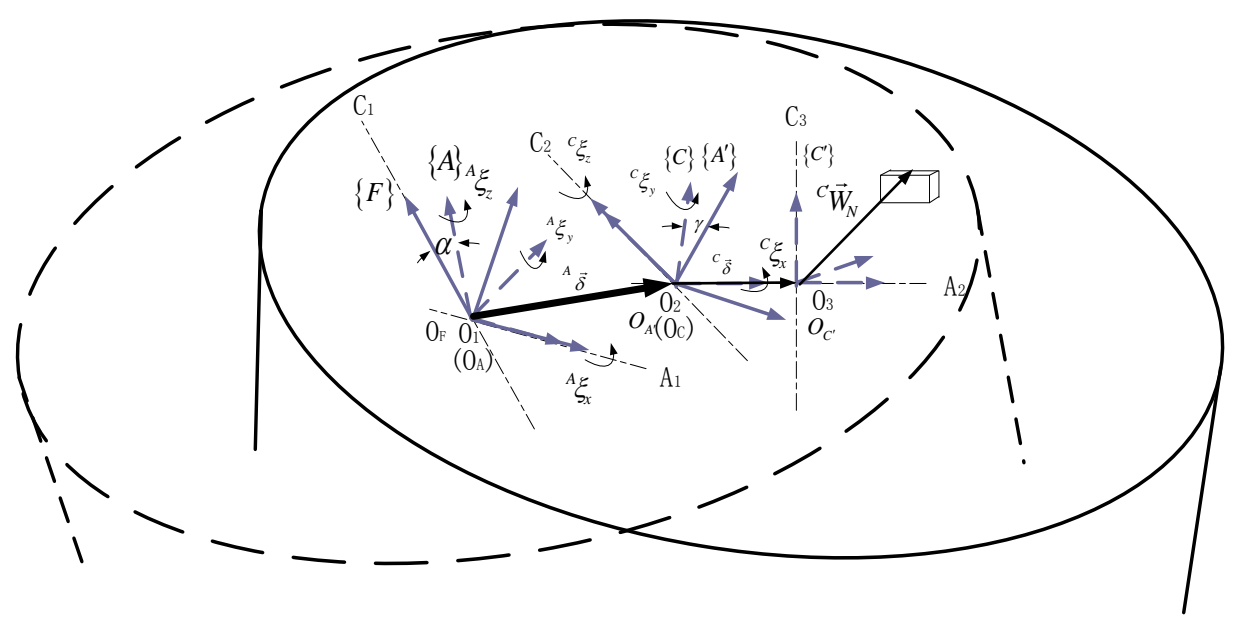

Figure.1 The kinematic model of the rotary table []

$$
\begin{aligned}
{ }_{A^{\prime}}^{A} R=\left[\begin{array}{ccc}
1 & -{ }^{A} \xi_{z} & { }^{A} \xi_{y} \\
{ }^{A} \xi_{z} & 1 & -{ }^{A} \xi_{x} \\
-{ }^{A} \xi_{y} & { }^{A} \xi_{x} & 1
\end{array}\right] \\
{ }_{C}^{A^{\prime}} R=\left[\begin{array}{ccc}
1 & 0 & 0 \\
0 & C \gamma & -S \gamma \\
0 & S \gamma & C \gamma
\end{array}\right]
\end{aligned}
$$

The initial position of the workpiece in the rotary table is:

$$
{ }^{C} \vec{W}_{N}=\left[\begin{array}{l}
0 \\
L \\
H
\end{array}\right]
$$

\section{Machining test 1}

Where, the distance of the workpiece from the original point in the direction of $+Y_{F}$ is $L$, and the value of $\mathrm{Z}$-axis of the machining plane is 0 .

To increase the error sensitivity, the workpiece should be fit on the table as far as possible from the centre point of the table. So the value of $\mathrm{L}$ should be as big as possible.

When $\alpha=90^{\circ}, \gamma=0^{\circ}$, cut the workpiece on the outer side along $+X_{\mathrm{F}}$ direction. When $\alpha=90^{\circ}, \gamma=180^{\circ}$, cut the workpiece on the outer side along $+\mathrm{X}_{\mathrm{F}}$ direction, too. The machining error between above two positions has the biggest error sensitivity to the kinematic errors in the virtue sensitivity direction.

According to the principle robotic kinematic, the positon vector of the workpiece in the reference coordinate system $\{\mathrm{F}\}$ can be obtained:

$$
{ }^{F} \vec{W}={ }_{A}^{F} R\left[{ }^{A} \vec{\delta}+{ }_{A^{\prime}}^{A} R_{C}^{A^{\prime}} R\left[{ }^{C} \vec{\delta}+{ }_{C^{\prime}}^{C} R^{C^{\prime}} \vec{W}_{N}\right]\right]
$$

Suppose that $\overrightarrow{\mathrm{r}}_{1}=\left[\begin{array}{lll}0 & -1 & 0\end{array}\right]$ is the virtue sensitive direction expressed as a unit vector. Suppose that:

When $\alpha=90^{\circ}, \gamma=180^{\circ}$, the center position of the workpiece is $\mathrm{Y}_{2}$.

When $\alpha=90^{\circ}, \gamma=0^{\circ}$, the center position of the workpiece is $\mathrm{Y}_{1} . h=\mathrm{Y}_{2^{-}} \mathrm{Y}_{1}$, we can obtain:

$$
h=-2 \cdot{ }^{A} \xi_{x}\left({ }^{C} \delta_{y}+L\right)+2 \cdot{ }^{A} \xi_{y} \cdot{ }^{C} \delta_{x}
$$




\footnotetext{
Where, the value of ${ }^{A} \xi_{y} \cdot{ }^{C} \delta_{x}$ is tiny and can be ignored. So formula (6) only include one kinematic error ${ }^{A} \xi_{x}$ of A-axis.
}

\section{Machining test 2}

The error-sensitive direction is $+Z_{F}$, and it can be represented by $r_{2}=\left[\begin{array}{lll}0 & 0 & 1\end{array}\right]$.

The position vector in the error-sensitive direction of $+Z_{\mathrm{F}}$ can be obtained:

$$
Z={ }^{F} \vec{W} \cdot\left[\begin{array}{lll}
0 & 0 & 1
\end{array}\right]
$$

When $\alpha=+90^{\circ}, \gamma=+180^{\circ}$, the center point in the surface of the workpiece in the direction of $-\mathrm{Y}_{\mathrm{F}}$ is $\mathrm{Z}_{2}$. When $\alpha=0^{\circ}, \gamma=0^{\circ}$, the center point in the surface of the workpiece in the direction of $-\mathrm{Y}_{\mathrm{F}}$ is $\mathrm{Z}_{1}$. We can obtain:

$$
Z_{2}-Z_{1}={ }^{F} \vec{W}_{1}\left(\alpha=90^{0}, \gamma=180^{0}\right) \cdot r_{3}-{ }^{F} \vec{W}_{1}\left(\alpha=0^{0}, \gamma=0^{0}\right) \cdot r_{3}
$$

Through calculation:

$$
\begin{aligned}
& \mathrm{Z}_{2}-\mathrm{Z}_{1}={ }^{A} \delta_{y}-\mathrm{L}-\mathrm{H}-{ }^{A} \delta_{z}-{ }^{C} \delta_{y}+\mathrm{H} \cdot{ }^{C} \xi_{x}-{ }^{C} \xi_{x} \cdot L-{ }^{A} \xi_{x} \cdot\left(H+L \cdot{ }^{C} \xi_{x}\right)+ \\
& { }^{A} \xi_{y} \cdot\left({ }^{C} \delta_{x}+H \cdot{ }^{C} \xi_{y}\right)-{ }^{A} \xi_{z} \cdot\left({ }^{C} \delta_{x}+H \cdot{ }^{C} \xi_{y}\right)-{ }^{A} \xi_{x} \cdot\left(L+{ }^{C} \delta_{y}-H \cdot C{ }^{C} \xi_{x}\right)
\end{aligned}
$$

There is a distance of $-\mathrm{L}$ in the direction of $+\mathrm{X}_{\mathrm{F}}$, and there is a distance of $-\mathrm{H}$ in the direction of $+\mathrm{Z}_{\mathrm{F}}$. Therefore, the actual machining error is

$$
\begin{aligned}
& \Delta l_{2}={ }^{A} \delta_{y}-{ }^{A} \delta_{z}-{ }^{C} \delta_{y}+\mathrm{H} \cdot{ }^{C} \xi_{x}-{ }^{C} \xi_{x} \cdot L-{ }^{A} \xi_{x} \cdot\left(H+L \cdot{ }^{C} \xi_{x}\right)+ \\
& { }^{A} \xi_{y} \cdot\left({ }^{C} \delta_{x}+H \cdot{ }^{C} \xi_{y}\right)-{ }^{A} \xi_{z} \cdot\left({ }^{C} \delta_{x}+H \cdot{ }^{C} \xi_{y}\right)-{ }^{A} \xi_{x} \cdot\left(L+{ }^{C} \delta_{y}-H \cdot{ }^{C} \xi_{x}\right)
\end{aligned}
$$

Where, the values of ${ }^{A} \xi_{y} \cdot\left({ }^{C} \delta_{x}+H \cdot{ }^{C} \xi_{y}\right)-{ }^{A} \xi_{z} \cdot\left({ }^{C} \delta_{x}+H \cdot{ }^{C} \xi_{y}\right),{ }^{A} \xi_{x} \cdot\left({ }^{C} \delta_{y}-H \cdot{ }^{C} \xi_{x}\right)$ and ${ }^{A} \xi_{x} \cdot{ }^{C} \xi_{x} \cdot L$ are tiny and can be ignored.

$$
\Delta l_{2}={ }^{A} \delta_{y}-{ }^{A} \delta_{z}-{ }^{C} \delta_{y}+(\mathrm{H}-\mathrm{L}) \cdot\left({ }^{C} \xi_{x}-{ }^{A} \xi_{x}\right)
$$

This machining error includes the kinematic errors of ${ }^{A} \xi_{x},{ }^{A} \vec{\delta}_{y}$ and ${ }^{A} \vec{\delta}_{z}$.

\section{Machining test 3}

The error-sensitive direction is $-Y_{F}$, it can be represented by the vector $r_{1}=\left[\begin{array}{ll}0-1 & 0\end{array}\right]$. The position vector of the workpiece in the direction of $-\mathrm{Y}_{\mathrm{F}}$ can be obtained:

$$
Z={ }^{F} \vec{W} \cdot\left[\begin{array}{lll}
0 & -1 & 0
\end{array}\right]
$$

When $\alpha=+90^{\circ}, \gamma=+180^{\circ}$, the value of the center point on the surface of the workpiece in the direction of $-\mathrm{Y}_{\mathrm{F}}$ is $\mathrm{Y}_{2}$. When $\alpha=0^{\circ}, \gamma=0^{\circ}$, the value of the center point on the surface of the workpiece in the direction of $-Y_{F}$ is $Y_{1}$. The difference of the position vector in the direction of $+Z_{F}$ between the workpice when the rotary table is moving and the workpice when the rotary table is stationary can be obtained:

$$
-\mathrm{Y}_{2}-\left(-\mathrm{Y}_{1}\right)={ }^{F} \vec{W}_{1}\left(\alpha=90^{\circ}, \gamma=180^{\circ}\right) \cdot r_{5}-{ }^{F} \vec{W}_{1}\left(\alpha=0^{\circ}, \gamma=0^{\circ}\right) \cdot r_{5}
$$

Through matrix calculation, we can obtain:

$$
\begin{aligned}
& -\mathrm{Y}_{2}-\left(-\mathrm{Y}_{1}\right)=\mathrm{H}+\mathrm{L}+{ }^{A} \delta_{y}+{ }^{A} \delta_{z}+{ }^{C} \delta_{y}-{ }^{C} \xi_{x} \cdot H+{ }^{C} \xi_{x} \cdot L-{ }^{A} \xi_{x} \cdot\left(H+{ }^{C} \xi_{x} \cdot L\right)+ \\
& { }^{A} \xi_{y} \cdot\left({ }^{C} \delta_{x}+{ }^{C} \xi_{y} \cdot H\right)+{ }^{A} \xi_{z} \cdot\left({ }^{C} \delta_{x}+{ }^{C} \xi_{y} \cdot H\right)-{ }^{A} \xi_{x} \cdot\left(L+{ }^{C} \delta_{y}-{ }^{C} \xi_{x} \cdot H\right)
\end{aligned}
$$

In the machining process, there is a distance of $\mathrm{L}$ of the tool in the distance of $+\mathrm{X}_{\mathrm{F}}$. and there is a distance of $\mathrm{H}$ of the tool in the distance of $+\mathrm{Z}_{\mathrm{F}}$. So the actual machining error is:

$$
\begin{aligned}
& \Delta l_{3}={ }^{A} \delta_{y}+{ }^{A} \delta_{z}+{ }^{C} \delta_{y}-{ }^{C} \xi_{x} \cdot H+{ }^{C} \xi_{x} \cdot L-{ }^{A} \xi_{x} \cdot\left(H+{ }^{C} \xi_{x} \cdot L\right)+ \\
& { }^{A} \xi_{y} \cdot\left({ }^{C} \delta_{x}+{ }^{C} \xi_{y} \cdot H\right)+{ }^{A} \xi_{z} \cdot\left({ }^{C} \delta_{x}+{ }^{C} \xi_{y} \cdot H\right)-{ }^{A} \xi_{x} \cdot\left(L+{ }^{C} \delta_{y}-{ }^{C} \xi_{x} \cdot H\right)
\end{aligned}
$$


Where, the values of ${ }^{A} \xi_{x} \cdot{ }^{C} \xi_{x} \cdot L,{ }^{A} \xi_{y} \cdot\left({ }^{C} \delta_{x}+{ }^{C} \xi_{y} \cdot H\right)+{ }^{A} \xi_{z} \cdot\left({ }^{C} \delta_{x}+{ }^{C} \xi_{y} \cdot H\right)$ and ${ }^{A} \xi_{x} \cdot\left({ }^{C} \delta_{y}-{ }^{C} \xi_{x} \cdot H\right)$ are very tiny and can be ignored. We can obtain:

$$
\Delta l_{3}={ }^{A} \delta_{y}+{ }^{A} \delta_{z}+{ }^{C} \delta_{y}+{ }^{C} \xi_{x} \cdot(L-H)-{ }^{A} \xi_{x} \cdot(H+L)
$$

This machining error includes the kinematic errors of ${ }^{A} \xi_{x},{ }^{A} \vec{\delta}_{y}$ and ${ }^{A} \vec{\delta}_{z}$

From formula (6), (11) and (16), ${ }^{A} \xi_{x}(\alpha), \quad{ }^{A} \vec{\delta}_{y}$ and ${ }^{A} \vec{\delta}_{z}$ can be obtained.

\section{Conclusions}

To identify the kinematic error of five-axis machine tool rotary table, several machining test paths were designed. The function relationship between the kinematic errors and the machining errors in the corresponding error sensitive direction was deduced, and 3 kinematic errors can be separated from the machining tests in theory. Actually, there may be several possible machining tests which are sensitive to one kinematic error. To improve the efficiency and precision of the kinematic errors identification, further analysis and experiment should be carried out to compare and choose a better machining test pattern.

\section{Acknowledgment}

This work was financially supported by Startup Foundation of Zhejiang University of Science and Technology (F701102H02).

\section{References}

[1] Y. Abbaszaheh-Mir, J. R. R. Mayer, G. Clotier, and C. Fortin, Theory and simulation for the identification of the link geometric errors for a five-axis machine tool using a telescoping magnetic ball bar. Int. J. of Production Research, 2002. 40(18): p. 4781-4797.

[2] S. Zargarbashi and J. Mayer, Assessment of machine tool trunnion axis motion error, using magnetic double ball bar. Int. J. of Machine Tools and Manufacture, 2006. 46(14): p.1823-1834.

[3] W. Lei, M. Sung, W. Liu, and Y. Chuang, Double ball bar test for the rotary axes of five-axis CNC machine tools. Int. J. of Machine Tools and Manufacture, 2007.47(2): p. 273-285.

[4] K. Dassanayake, C. Cui and M. Tsutsumi, Accuracy evaluation method for multi-tasking turning centre. Int. J. Mechatronics and Manufacturing Systems, 2011.4(3/4): p. 285-303.

[5] Yukitoshi Thara, Ball Bar Measurement on Machine Tools with Rotary Axes. Int. J. of Automation Technology, 2012.6(2): p. 180-187.

[6] B. Bringmann and W. Knapp, Model-based 'Chase-the-Ball' calibration of a 5-axis machining center. CIRP Annals - Manufacturing Technology, 2006. 55(1): p.531-534.

[7] Weikert, S., R-test, a new device for accuracy measurements on five axis machine tools. Cirp Annals-Manufacturing Technology, 2004. 53(1): p. 429-432.

[8] ZHANG Ya, FU Jianzhong, CHEN Zichen. Machining tests to identify kinematic errors of machine tool table rotation axis based on sensitive directions [J]. The International Journal of Advanced Manufacturing Technology, 2013, 67(1-4): 495-500.

[9] Xiong Youlun (1993) Robotics. China Machine Press, Beijing 